Article

\title{
Investigation of the Moderating Effect of Culture on Users' Susceptibility to Persuasive Features in Fitness Applications
}

\author{
Kiemute Oyibo $* \mathbb{B}$ and Julita Vassileva \\ Department of Computer Science, University of Saskatchewan, Saskatoon, SK S7N 5C9, Canada; jiv@cs.usask.ca \\ * Correspondence: kiemute.oyibo@usask.ca
}

Received: 8 September 2019; Accepted: 31 October 2019; Published: 6 November 2019

\begin{abstract}
Persuasive technologies have been identified as a potential motivational tool to tackle the rising problem of physical inactivity worldwide, with research showing they are more likely to be successful if tailored to the target audience. However, in the physical activity domain, there is limited research on how culture moderates users' susceptibility to the various persuasive features employed in mobile health applications aimed to motivate behavior change. To bridge this gap, we conducted an empirical study among 256 participants from collectivist $(n=67)$ and individualist ( $n=189)$ cultures to determine their culture-specific persuasion profiles with respect to six persuasive features commonly employed in fitness applications on the market. The persuasive features include two personal features (goal-setting/self-monitoring and reward) and four social features (competition, cooperation, social learning and social comparison). We based our study on the rating of storyboards (on which each of the six persuasive features is illustrated) and the ranking of the six persuasive features in terms of perceived persuasiveness. The results of our analysis showed that users from individualist and collectivist cultures significantly differ in their persuasion profiles. Based on our rating measure, collectivist users are more likely to be susceptible to all six persuasive features (personal and social) than individualist users, who are only likely to be susceptible to personal features. However, based on our ranking measure, individualist users are more likely to be susceptible to personal features (goal-setting/self-monitoring and reward) than collectivist users. In contrast, collectivist users are more likely to be susceptible to social features (cooperation and social learning) than individualist users. Based on these findings, we provide culture-specific persuasive technology design guidelines. Our study is the first to uncover the moderating effect of culture on users' susceptibility to commonly employed persuasive features in fitness applications.
\end{abstract}

Keywords: persuasive technology; fitness application; persuasive features; culture; goal-setting; self-monitoring; reward; competition; cooperation; social learning; social comparison; tailoring

\section{Introduction}

Fitness applications are gaining traction worldwide due to the global problem of physical inactivity and the need to become fit and/or stay in shape by exercising regularly. These applications are being used in different contexts (at home, in the gym, in the hotel, etc.) to motivate exercise behavior change. They are also being used in different modes (personal and social) to encourage exercise behavior change. However, for these applications to be more effective, there is a need for designers to tailor them to the target audience. Research [1-3] shows that understanding potential users' persuasion profiles will help designers tailor persuasive health applications to future users to make them more effective. Persuasion profile [4] is an ordered list of persuasive strategies or features (in terms of perceived persuasiveness) to which potential users of a persuasive health application are likely to be susceptible. 
Moreover, research shows that persuasive applications can be tailored based on demographic variables. In particular, gender $[5,6]$ and culture $[7,8]$ have been found to be useful demographic factors based on which persuasive health applications can be tailored to different user groups in a given population. Group-based tailoring has become important given that, in certain situations, designers may not have the required resources (time, money and/or programming expertise) to implement fine-grain tailoring (aka personalization) at the personal level, which entails tailoring to the individual based on his/her personal dynamic user model and profile. In such a case, while individual-based tailoring could be easily implemented by big players (such as Samsung, Google, Apple, Fitbit, etc.) given the huge human and algorithmic resources at their disposal, for small players with limited resources, tailoring at the group level may be necessary to reduce the cost of application implementation and deployment time to market.

However, in the health domain, there are limited cross-cultural studies aimed at investigating how different cultural groups may respond to different persuasive strategies employed in motivating exercise behavior change. Specifically, there are limited comparative studies involving participants from Western and African cultures. Most persuasive health applications are designed and targeted at users in Western cultures. As such, most persuasive technology studies have been focused on Western societies in North America and Europe [9]. However, most persuasive health applications are used in non-Western societies in Asia and Africa as well. While the two major target countries in North America (United States and Canada) are individualist cultures, most target countries in Asia (e.g., China) and Africa (e.g., Nigeria) are collectivist cultures. Individualist cultures are societies in which people are independent and self-motivated in their pursuits of life's goals, while collectivist cultures are societies in which people are inter-dependent and socially motivated in the pursuit of life's goals. Research [7] shows that this difference in the two main types of cultures influences the way people in the respective cultures perceive and use human-computer interaction (HCI) applications in general and persuasive technologies in particular. Hence, persuasive strategies that are effective in Europe/North America may not be effective in Asia and/or Africa. For this reason, there have been several calls on the need to tailor persuasive applications to different cultures to make them more effective (e.g., [10]). However, in the fitness domain, there is limited cross-cultural research on the differences between users from individualist and collectivist cultures in terms of their susceptibility to persuasive strategies commonly employed in mobile health applications. According to [11], examining how culture influences persuasion has not yet been given much attention by persuasive technology researchers. Designers can leverage the empirical evidence on cultural differences to tailor persuasive health applications to users in the respective cultures so that they can be more effective in motivating exercise behavior change.

As a result, we conducted an empirical study to investigate how subjects from Canada/United States (individualist culture) and Nigeria (collectivist culture) differ with respect to their susceptibility to six commonly employed persuasive strategies in fitness applications on the market. They include goal-setting/self-monitoring, reward, competition, cooperation, social learning and social comparison [12,13]. These strategies were illustrated on storyboards as persuasive features and participants from both cultures were requested to rate them in terms of perceived persuasiveness. Secondly, participants were asked to rank all six persuasive strategies from the one that would motivate them the most to the one that would motivate them the least. The ranking measure was employed in addition to the rating measure to offset the different levels of criticality that may exist in the evaluation (rating) of human-computer interaction (HCI) artifacts (e.g., [14]) by subjects from both cultures.

The results of our analysis revealed that, based on the ratings of the storyboards, participants from the collectivist culture are likely to be susceptible to all six (personal and social) persuasive features. However, participants from individualist culture are only likely to be susceptible to the personal persuasive features (goal-setting/self-monitoring and reward). The only social strategy individualist participants are likely to be susceptible to is competition. Our between-group analysis shows that, based on the rating of the six storyboards, the collectivist group is more likely to be susceptible to 
all six persuasive features than the individualist group $(p<0.05)$. However, based on the ranking of the six persuasive strategies-which is independent of the different levels of criticality that may exist in the evaluation of $\mathrm{HCI}$ artifacts by both cultures-our results show that the individualist group is more likely to be susceptible to goal-setting/self-monitoring and reward than the collectivist group $(p<0.05)$. On the other hand, our results revealed that the collectivist group is more likely to be susceptible to cooperation and social learning than the individualist group $(p<0.05)$. Thus, we conclude that, overall, personal persuasive strategies (such as goal-setting/self-monitoring and reward) are more likely to be effective in individualist cultures than in collectivist cultures, while social persuasive strategies (cooperation and social learning) are more likely to be effective in collectivist cultures than in individualist cultures. Moreover, we conclude that both personal and social persuasive strategies are likely to be effective in collectivist cultures such as Nigeria. However, only competition, in addition to the personal strategies, are likely to be effective in individualist cultures such as Canada/United States. Thus, we recommend that, while personal and social persuasive strategies can be employed to motivate behavior change in collectivist cultures, personal persuasive strategies should be given priority in individualist cultures.

The rest of the paper is organized as follows. Section 2 provides a background on persuasive strategies/features. Section 3 dwells on the related work. Section 4 describes the study design and method. Section 5 focuses on the result. Section 6 presents the discussion, while Section 7 concludes the paper.

\section{Background}

This section provides a brief overview of the cultural framework used in this study as an analytic framework of comparative analysis and the six persuasive features commonly employed in persuasive health applications.

\subsection{Culture}

Culture is defined as the collective programming of the mind [15], which results in a common way of doing things (e.g., speaking, dressing, eating, etc.) by a group of people in the larger society. In HCI, research [16] shows that culture can influence the way people perceive and interact with information systems and artifacts such as websites [8], health applications [10], etc. Hofstede's cultural framework (individualism vs. collectivism) turns out to be one of the most widely used frameworks for HCI research (e.g., [7]). Individualism is a worldview that puts the "I" and "Me" before the "We" and "Us". Hence, in the individualist culture, people are more concerned about the pursuit of personal goals than collective goals. Thus, they become very independent, self-reliant and self-motivated in the pursuit and achievement of their personal goals and aspirations. In contrast, collectivism is a worldview that puts the "We" and "Us" before the "I" and "Me". Hence, in the collectivist culture, people are more concerned about the pursuit of social goals than personal goals. Thus, they become very interdependent and socially driven in the achievement of the goals and aspirations of the in-groups to which they belong. In this paper, our comparative analysis was conducted using this cultural framework of individualism vs. collectivism using Canada/United States and Nigeria, respectively, as a case study.

\subsection{Persuasive Features}

Persuasive features are motivational affordances with which persuasive applications are equipped to promote user adoption and behavior change [17]. Table 1 shows the definition of all six persuasive features of interest, which are commonly employed in persuasive health applications on the market to motivate behavior change [12]. They are drawn from the persuasive system design (PSD) model put forward by Oinas-Kukkonen and Harjumaa [18]. 
Table 1. Commonly employed persuasive features in persuasive applications and their definitions $[12,13,19,20]$. The "*" indicates that, in our storyboards, we implemented goal-setting and self-monitoring as a single feature as we considered both as complementary features and discussed them as such in the paper.

\begin{tabular}{cl}
\hline Feature & \multicolumn{1}{c}{ Definition } \\
\hline Goal-Setting * & $\begin{array}{l}\text { Goal-setting is a persuasive feature that allows users to set goals prior to tracking their } \\
\text { performance of the target behavior and progress over time. }\end{array}$ \\
\hline Self-Monitoring * & $\begin{array}{l}\text { Self-monitoring is a persuasive feature that allows users to keep track of their performance of } \\
\text { the target behavior and progress over time. }\end{array}$ \\
\hline Reward & $\begin{array}{l}\text { Reward is a persuasive feature that allows incentives such as points, badges, levels, etc., to be } \\
\text { awarded to users whenever they achieve their goals or reach a certain milestone. }\end{array}$ \\
\hline Cooperation & $\begin{array}{l}\text { Cooperation is a persuasive feature that allows two or more users to work together as a team } \\
\text { to reach their collective goal. }\end{array}$ \\
\hline Social Learning & $\begin{array}{l}\text { Social learning is a persuasive feature that allows users in a collaborative setting to observe } \\
\text { the performance of the behavior, progress and achievements of one another. }\end{array}$ \\
\hline Competition & $\begin{array}{l}\text { Social comparison is a persuasive feature that allows users in a collaborative setting to view } \\
\text { and compare their performance, progress and achievements with those of others. }\end{array}$ \\
\hline $\begin{array}{l}\text { Competition is a persuasive feature that allows users to compete against one another } \\
\text { towards achieving a mutually exclusive reward. }\end{array}$ \\
\hline
\end{tabular}

\section{Related Work}

In the persuasive technology domain, a substantial amount of studies has been conducted to uncover user susceptibilities to persuasive strategies with a view to tailoring persuasive applications to different target populations. Such studies usually look at gender, age and culture as potential demographic variables based on which persuasive applications can be tailored to users to make them more effective. In this review, we focused on the relevant studies.

Kaptein et al. [21] conducted a study to examine the relationship between users' susceptibility to persuasive cues and compliance to persuasive requests. They found that the susceptibility to persuasive cues could enhance users' compliance to persuasive requests. Specifically, they found that the more susceptible users are to persuasive cues, the more likely they will comply with persuasive requests such as provision of email address. However, the authors did not investigate the influence of culture on users' compliance to persuasive requests, neither did they conduct the study in the physical activity domain.

Orji et al. [22,23] examined the effect of culture, gender and age on users' susceptibility to the six Cialdini's principles of persuasion [24] using subjects from North America and Asia as a case study. The Cialdini's [24] six principles include commitment, reciprocity, authority, liking, consensus and scarcity. Orji et al. $[22,23]$ found that, irrespective of all three demographic factors, users are most likely to be responsive to the commitment principle, which is usually mapped to goal-setting in the persuasive technology domain [25]). However, in the first study, Orji et al. [23] found that adults are more likely to be susceptible to commitment than younger adults, while younger adults are more likely to be susceptible to scarcity than adults [23]. In the same study, the authors found that females are more likely to be susceptible to commitment, reciprocity and consensus than males [23]. In the second study, Orji [22] found that users from collectivist culture are more likely to be susceptible to authority, reciprocity, liking and consensus than users from individualist cultures. However, the authors did not investigate most of the persuasive strategies our current study is concerned with, such as reward, competition, social learning, cooperation, etc.

As a follow-up to Orji et al.'s [22,23] studies, Oyibo et al. [25,26] carried out similar studies to examine the effect of culture [25] and gender [26] on users' susceptibility to all six Cialdini's principles of persuasion using subjects from Canada (individualist culture) and Nigeria (collectivist culture) as a case study. The authors replicated Orji et al.'s [22,23] findings. Specifically they found that, regardless of culture and gender, participants are most [25] likely to be susceptible to the commitment principle. Regarding the effect of culture, Oyibo et al. [25] found that individualists are more likely to 
be susceptible to reciprocity, liking and consensus than collectivist, while collectivists are more likely to be susceptible to authority than individualists. All of these findings, which were based on the rating measure, were replicated using the ranking measure, except that regarding reciprocity. While Oyibo et al.'s [25] study among collectivists (Nigerians) and individualists (Canadians) replicated Orji's [22] finding that collectivists (Asians) are more likely to be susceptible to authority than individualists (North Americans), it did not regarding reciprocity and liking. Orji [22] found that collectivists (Asians) are more likely to be susceptible to reciprocity and liking than individualists (North Americans). However, Oyibo et al. [25] found that individualists (Canadians) are more likely to be susceptible to reciprocity and liking than collectivists (Nigerians). This finding suggests that collectivists from different continents and countries could differ in their susceptibility to Cialdini's principles of persuasion Therefore, more studies need to be done in this research area to investigate how collectivists in different continents and countries (e.g., Africa vs. Asia, Nigeria vs. China, etc.) differ in their responsiveness to Cialdini's principles of persuasion in particular and persuasive strategies in general. However, Oyibo et al.'s [25] study, just like Orji et al.'s [22,23] studies, did not investigate persuasive strategies such as reward, competition, social learning, cooperation, etc., which our present study addressed. Oyibo et al.'s [25] study was also not carried out in the specific context of physical activity as our current study.

Oyibo et al. [27] investigated the moderating effect of culture on the influence of gender and age on social-influence strategies (reward, competition, social comparison and social learning) using Nigeria (collectivist culture) and Canada (individualist culture) as a case study. They found that males are more likely to be susceptible to reward and competition than females in the collectivist culture, but no difference between both genders in the individualist culture. They also found that younger people are more likely to be susceptible to social comparison and social learning than older people in the collectivist culture but no difference between both age groups in the individualist culture. Moreover, they found that younger people are more likely to be susceptible to competition than older people in both cultures. However, the authors did not investigate how both types of cultures differ directly with respect to their susceptibility to the social-influence strategies they investigated. In addition, their study was not carried out in the physical activity domain; it was done in a non-domain-specific context.

Finally, in our earlier studies, we investigated the susceptibility of collectivists (residents in Nigeria) [28] and individualists (resident in Canada/United States) [17] to all six persuasive features we examined in this paper (goal-setting/self-monitoring, reward, competition, cooperation, social learning and social comparison). In the first study [28], we found that, based on the rating measure, collectivists are likely to be susceptible to all six features. In particular, we found that they are most likely to be susceptible to goal-setting/self-monitoring and reward and least likely to be susceptible to social comparison and social learning. However, based on the ranking measure, we found that they are more likely to be susceptible to goal-setting/self-monitoring than the other persuasive features. In the second study [17], we found that, based on the rating measure, individualists are likely to be susceptible to the personal features (goal-setting/self-monitoring and reward) and competition, and unlikely to be susceptible to cooperation, social learning and social comparison. These findings were replicated using the ranking measure. However, in both studies, we did not investigate the moderating effect of culture. Our current study aimed to bridge this gap by comparing how users in collectivist and individualist cultures differ in their susceptibility to the six persuasive features of interest, which are commonly employed in persuasive applications aimed at motivating behavior change.

\section{Method}

This section focuses on the research objective, measurement instruments and demographics of the participants that took part in the study.

\subsection{Research Objective}

The aim of our study is to investigate, using storyboards, the differences between the individualist culture and the collectivist culture with respect to their persuasion profiles [4] using Canada/United 
States and Nigeria, respectively, as a case study. The persuasion profiles are based on six commonly employed persuasive features (goal-setting/self-monitoring, reward, competition, cooperation, social learning and social comparison) illustrated on storyboards. These persuasive features are drawn from the PSD model [18]. For example, Figure 1 shows the storyboard for social learning. In this storyboard, the user is able to view the goal of his friend, Kim, who sets out to achieve a small goal of 4000 calories for the day. Once Kim achieves his goal, the user receives a notification informing him that Kim has achieved his goal. Storyboards such as this have been successfully used in prior studies [12] to uncover useful persuasive system design information from potential users. Using storyboards such as these, the main research question this study aimed to address is presented as follows:

"What are the differences between the persuasion profiles of users in collectivist and individualist cultures with respect to their susceptibilities to goal-setting/self-monitoring, reward, competition, cooperation, social learning and social comparison?"

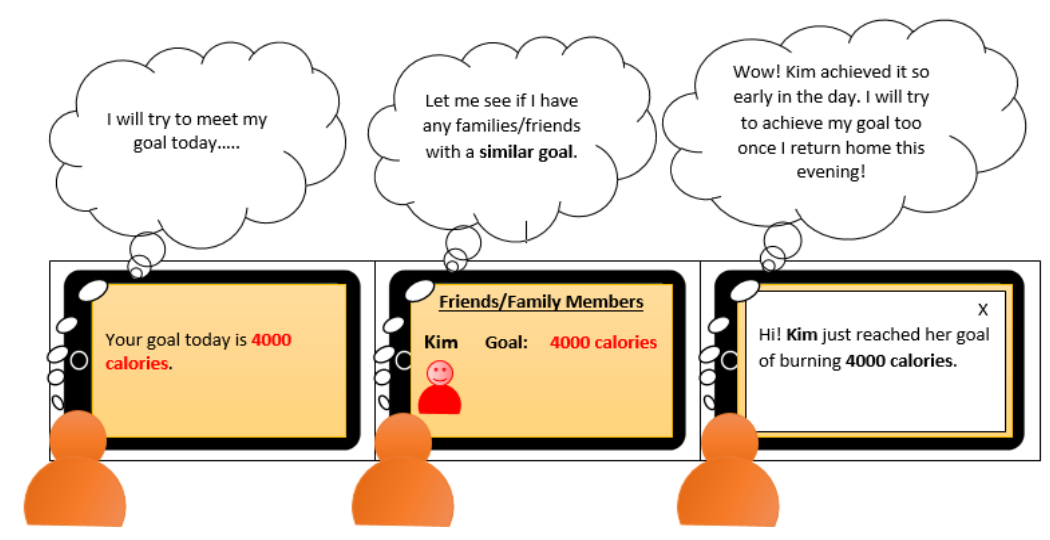

Figure 1. Storyboard illustrating a social learning persuasive feature [17].

\subsection{Research Design and Measurement Instruments}

The research design was based on the rating and ranking of the six persuasive features of interest. In an online survey, six storyboards illustrating the six persuasive features were administered to participants from both cultures. This was followed by: (1) a questionnaire on the perceived persuasiveness of the features illustrated on the storyboards for participants to answer; and (2) a list of the six persuasive features for participants to rank in terms of perceived persuasiveness. To contextualize the study and set the tone for the participants' response to the questionnaire, we presented them with a description of a hypothetical fitness application we called the "Homex App". The description read as follows:

"Imagine you want to improve your personal health and fitness level. Given the challenges (e.g., time, cost, weather, etc.) associated with going to the gym regularly, the "Homex App" has been created, say by health promoters in your neighborhood, to support your physical activity."

\subsubsection{Rating Measure}

Regarding the rating measure, the following question (together with the items from the perceived persuasiveness scale [29]) was posed to participants after each of the storyboards (e.g., Figure 1) had been shown to them:

"Imagine that you are using the Homex App presented in the storyboard above (see Figure 1) to track your physical activity, to what extent do you agree with the following statements:

1. This feature of the app would influence me.

2. This feature of the app would be convincing.

3. This feature of the app would be personally relevant to me.

4. This feature of the app would make me reconsider my physical activity." 
The above scale—ranging from "Strongly Disagree (1)" to "Strongly Agree (7)" —has been used in prior studies such as [2]. Before participants began answering the above questions, they were asked to briefly study the persuasive feature being illustrated on each storyboard and choose the correct one from a list of options. Each option comprised the name and definition of each persuasive feature. For example, goal-setting/self-monitoring was defined as "an application that allows a user to set a goal and track his/her own performance or status. It provides information on both past and current performance". This was intentionally done to increase the reliability of participants' responses and our findings. As such, in the process of data cleaning, participants' responses to wrongly identified persuasive features were treated as missing data points and filled in with the appropriate average scores.

\subsubsection{Ranking Measure}

In addition to rating the six persuasive features illustrated on the storyboards, participants were asked to rank them as well. The persuasive features were presented to participants in a list format, all of which were randomized for each participant. We added the ranking measure to account for the different levels of criticality that may exist between the individualist and collectivist cultures when evaluating HCI artifacts. For example, Oyibo et al. [14] found that Canadians (who are from an individualist culture) are more likely to be critical of mobile website design than Nigerians (who are from a collectivist culture). To account for this cultural difference, we asked participants to rank the six persuasive features (names only) from the one that would be most helpful to the one that would be least helpful in achieving their goals. The question we asked them is, "Please rank these features, starting with the one you think will help you achieve your exercise goals the most (FIRST) to the least (SIXTH)". This question will help us to tease out which set of persuasive features to which one culture is more likely to be susceptible than the other. We might not be able to tease out this information based on the rating measure alone if the rating of the persuasive features illustrated on the storyboards was influenced by the user-interface (UI) and information design. For example, one culture, overall, may rate their susceptibility to the persuasive features illustrated on the storyboards higher than the other by taking into consideration their judgment of the UI and content design.

\subsection{Participants}

Our study was submitted to the Behavioral Research Ethics Board of our university for review. Upon approval, we posted it on the Amazon Mechanical Turk (AMT) platform to recruit participants resident in Canada and United States and used email to recruit participants resident in Nigeria. Table 2 shows the demographics of the valid participants. The culture-based categorization was based on Hofstede's cultural framework [30]. A participant is categorized as an individualist if $\mathrm{s} / \mathrm{he}$ is resident in and has his/her country of origin as Canada/United States. On the other hand, a participant is categorized as a collectivist if $\mathrm{s} /$ he is resident in Nigeria and has his/her country of origin as Nigeria. Overall, a total of 315 participants took part in the study ( 87 collectivists and 228 individualists). After cleaning the data, we were left with 67 collectivist participants and 189 individualist participants. Each participant from Canada/United States was compensated with US $\$ 1.50$, while each participant from Nigeria was compensated with N200 credit card. Specifically, we recruited participants from Nigeria via email because very few Nigerians use the AMT platform compared with the larger number of Canadians and Americans that use the platform. 
Table 2. Demographics of participants. COL $=$ Collectivist culture, IND = Individualist culture.

\begin{tabular}{|c|c|c|c|c|c|}
\hline \multirow[b]{2}{*}{ Criterion } & \multirow[b]{2}{*}{ Subgroup } & \multicolumn{2}{|c|}{ Number (\#) } & \multicolumn{2}{|c|}{ Percent (\%) } \\
\hline & & COL & IND & $\mathrm{COL}$ & IND \\
\hline \multirow{3}{*}{ Gender } & Female & 29 & 82 & 43.3 & 43.4 \\
\hline & Male & 35 & 106 & 52.2 & 56.1 \\
\hline & Other & 3 & 1 & 4.5 & 0.5 \\
\hline \multirow{6}{*}{ Age } & $18-24$ & 26 & 29 & 38.8 & 15.3 \\
\hline & $25-34$ & 29 & 100 & 43.3 & 52.9 \\
\hline & $35-44$ & 9 & 39 & 13.4 & 20.6 \\
\hline & $45-54$ & - & 15 & - & 7.9 \\
\hline & $54+$ & - & 6 & - & 3.2 \\
\hline & Unspecified & 3 & - & 4.5 & - \\
\hline \multirow{6}{*}{ Education } & $\begin{array}{l}\text { Technical/Trade } \\
\text { School }\end{array}$ & 1 & 37 & 1.5 & 19.6 \\
\hline & High School & 2 & 34 & 3.0 & 18.0 \\
\hline & Bachelor & 51 & 86 & 76.1 & 45.5 \\
\hline & Masters & 10 & 26 & 14.9 & 13.8 \\
\hline & Doctorate & - & 4 & & 2.1 \\
\hline & Others & 3 & 2 & 4.5 & 1.1 \\
\hline \multirow{3}{*}{$\begin{array}{l}\text { Country of } \\
\text { Origin }\end{array}$} & Canada & - & 89 & - & 47.1 \\
\hline & United States & - & 100 & - & 52.9 \\
\hline & Nigeria & 67 & - & 100.0 & - \\
\hline \multirow{5}{*}{ Occupation } & Employee & 34 & 110 & 50.7 & 58.2 \\
\hline & Employer & 5 & 7 & 7.5 & 3.7 \\
\hline & Self-employed & 6 & 38 & 9.0 & 20.1 \\
\hline & Student & 14 & 26 & 20.9 & 13.8 \\
\hline & Other & 8 & 8 & 11.9 & 4.2 \\
\hline \multirow{6}{*}{$\begin{array}{l}\text { Years on the } \\
\text { Internet }\end{array}$} & $1-5$ & 9 & 1 & 13.4 & 0.5 \\
\hline & $6-10$ & 30 & 28 & 44.8 & 14.8 \\
\hline & $11-15$ & 18 & 39 & 26.9 & 20.6 \\
\hline & $16-20$ & 7 & 66 & 10.4 & 34.9 \\
\hline & $20+$ & - & 55 & & 29.1 \\
\hline & Unspecified & 3 & - & 4.5 & - \\
\hline
\end{tabular}

\section{Results}

This section covered the results of our data analysis, including the reliability analysis for the six persuasive constructs, their average ratings and rankings and analysis of variance (ANOVA).

\subsection{Measurement Instrument Reliability Analysis}

Prior to conducting data analyses (computation of mean values of persuasive features and analysis of variance), we carried out reliability tests on the six persuasive strategies (constructs) to ensure they were reliably measured by their respective scales. Given that our data set was not normally distributed like most questionnaire data, we based the reliability tests on the McDonald's omega $(w)$ metric rather than the Cronbach's alpha $(\alpha)$ metric, which is meant for normally distributed data [31]. Specifically, we used the "ci.reliability" function in the "MBESS" library in R for the reliability test for each construct. The results of the analyses showed that all of the constructs met the reliability requirement (i.e., $\omega \geq 0.7$ ) [32]. 


\subsection{Overall Average Rating and Ranking of Persuasive Features}

To determine the overall persuasion profile (i.e., level of susceptibility of participants to the six persuasive features of interest), we calculated the mean rating and ranking of each persuasive feature as shown in Figure 2. If we were to use the mean-rating values above the neutral value of 4 (on a 1-7 Likert scale) as an indication of susceptibility likelihood, then, overall, based on the rating metric, participants were more likely to be susceptible to goal-setting/self-monitoring $(\mathrm{M}=4.86)$, reward $(\mathrm{M}=4.37)$ and competition $(\mathrm{M}=4.26)$ and less likely to be susceptible to cooperation $(\mathrm{M}=3.78)$, social comparison $(\mathrm{M}=3.81)$ and social learning $(\mathrm{M}=3.92)$ Similarly, if we were to use the mean-ranking values above the overall average value of 3.5 (on a 1-6 ranking scale) as an indication of susceptibility likelihood, then, overall, based on the ranking metric, participants were more likely to be susceptible to goal-setting/self-monitoring $(\mathrm{M}=4.37)$ and reward $(\mathrm{M}=4.87)$ and less likely to be susceptible to social comparison $(\mathrm{M}=2.75)$, cooperation $(\mathrm{M}=3.08)$, social learning $(\mathrm{M}=3.10)$ and competition $(\mathrm{M}=3.40)$. Overall, the rating and ranking profiles seemed to correspond. For example, based on the rating and ranking metrics, goal-setting/self-monitoring turned out to be the most persuasive feature, followed by reward and competition. This is an indication that the determination of the persuasion profile of a given population could be based on ranking of persuasive features instead of rating.
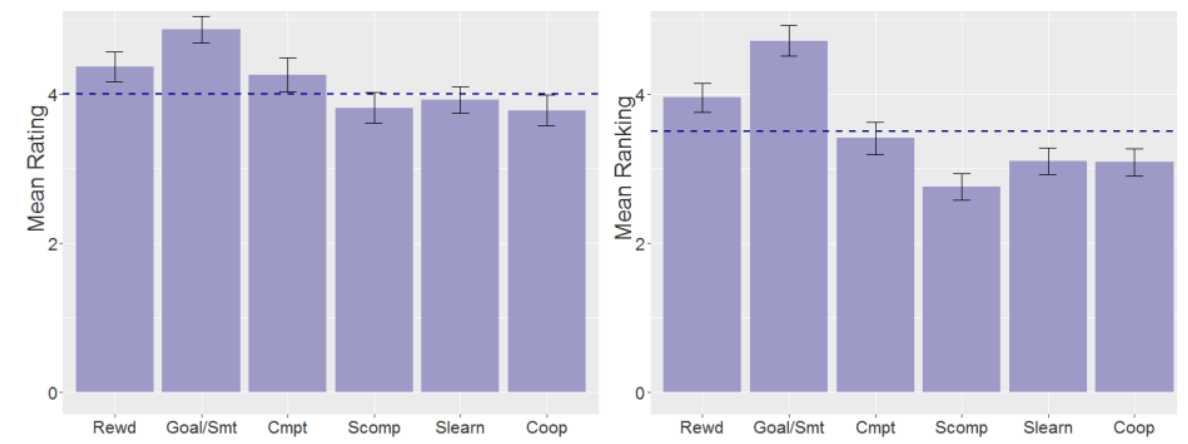

Figure 2. Overall mean rating (left) and ranking (right) of persuasive features. Crossbar indicates the neutral value on the 1-7 Likert scale and the mean value on the 1-6 ranking scale; REWD = Reward, GOAL/SMT = Goal-Setting/Self-Monitoring, CMPT = Competition, SCOMP = Social Comparison, SLEARN = Social Learning, $\mathrm{COOP}=$ Cooperation .

\subsection{Culture-Based Average Rating and Ranking of Persuasive Features}

Figure 3 shows the culture-specific mean ratings and rankings, respectively, of the six persuasive features. The barcharts reveal that, for the collectivist group, they are likely to be susceptible to all six persuasive features (based on the rating measure) and to goal-setting/self-monitoring, cooperation, social learning and reward (based on the ranking measure). On the other hand, for the individualist group, the barcharts show that they are likely to be susceptible to goal-setting/self-monitoring, reward and competition (based on the rating and ranking measures).

Comparatively, based on the rating measure, the barcharts reveal that the collectivist group is more likely to be susceptible to the six persuasive features than the individualist group. However, based on the ranking measure, the barcharts reveal that the individualist group is more likely to be susceptible to goal-setting/self-monitoring and reward than the collectivist group, while the collectivist group is more likely to be susceptible to cooperation and social learning than the individualist group. That said, our two-way ANOVA will determine whether, for each measure and persuasive feature, the numerical difference in the mean scores of the two cultural groups are statistically significant at $p<0.05$. 

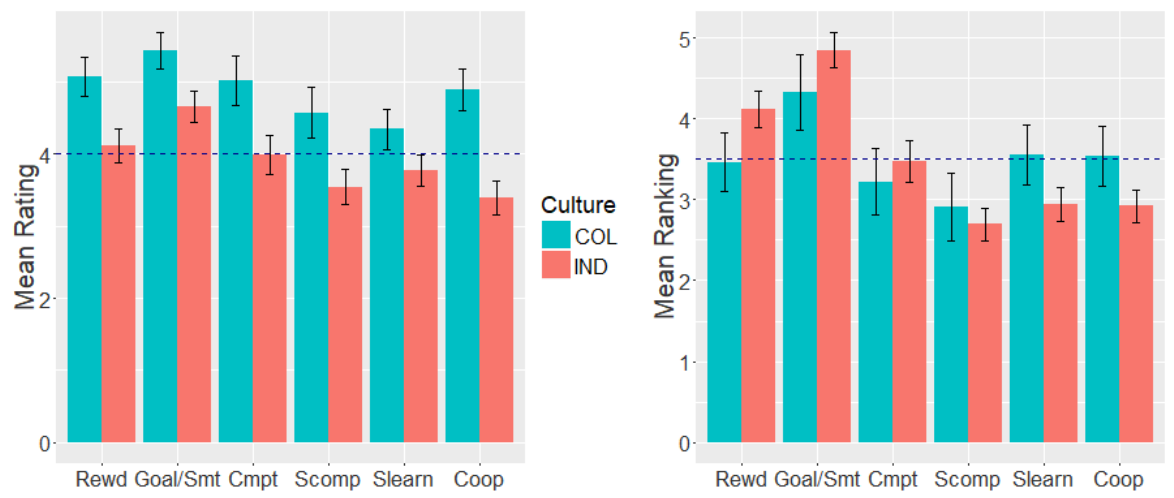

Figure 3. Culture-based mean rating (left) and ranking (right) of persuasive features. Crossbar indicates the neutral value on the 1-7 Likert scale and the mean value on the 1-6 ranking scale; REWD = Reward, GOAL/SMT = Goal-Setting/Self-Monitoring, CMPT = Competition, SCOMP = Social Comparison, SLEARN = Social Learning, $\mathrm{COOP}=$ Cooperation; $\mathrm{COL}=$ Collectivist culture, IND = Individualist culture.

\subsection{Two-Way Analysis of Variance (ANOVA) Based on Culture and Persuasive Feature}

Given the non-normality of our rating- and ranking-based data, we conducted a non-parametric repeated measure ANOVA $[33,34]$ using the ARTool package in $\mathrm{R}[35]$. With respect to the rating measure, the result of our analysis shows that there was a main effect of culture $\left(\mathrm{F}_{1}, 1524=117.42\right.$, $p<0.001)$, main effect of persuasive feature $\left(\mathrm{F}_{5,1524}=19.30, p<0.001\right)$ and an interaction between culture and persuasive feature $\left(\mathrm{F}_{5,1524}=2.89, p<0.05\right)$. Moreover, with respect to the ranking measure, there was no main effect of culture $\left(\mathrm{F}_{1}, 1524=0.18, p=\mathrm{n} . \mathrm{s}\right)$. However, there was a main effect of the persuasive feature $\left(\mathrm{F}_{5,1524}=40.53, p<0.001\right)$ and an interaction between culture and persuasive feature $\left(\mathrm{F}_{5,1524}=6.04, p<0.001\right)$. Due to the interaction between the culture and persuasive feature, we proceeded to conduct between-group and within-group analyses.

\subsubsection{Culture Effect Based on Between-Group Comparison}

Table 3 shows the results of the between-group analysis based on the rating and ranking measures. The rating-based result shows a significant difference between both cultural groups with respect to all six persuasive features. Specifically, the result shows that the collectivist group was significantly more likely to be susceptible to all six persuasive features $(p<0.05)$. However, the ranking-based result shows that the individualist group was more likely to be susceptible to reward $(p<0.05)$ than the collectivist group, while the collectivist group was more likely to be susceptible to cooperation and social learning than the individualist group $(p<0.01)$. Similarly, the ranking-based result shows that the individualist group was more likely to be susceptible to goal-setting/self-monitoring. However, the $p$-value of the between-group statistical significance test $(p=0.085)$ was marginal. Thus, more research needs to be done to investigate this marginal difference between both cultural groups with respect to goal-setting/self-monitoring. 
Table 3. Between group comparisons based on a non-parametric Kruskal-Wallis test. COL $=$ Collectivist culture, IND = Individualist culture; the bolded values indicate there is a significant difference $(p<0.05)$ between collectivist and individualist scores.

\begin{tabular}{ccccccccc}
\hline \multicolumn{3}{c}{ Rating Measure } & \multicolumn{4}{c}{ Ranking Measure } \\
\hline Feature & Overall & COL & IND & $\boldsymbol{p}$-Value & Overall & COL & IND & $p$-Value \\
\hline REWD & 4.37 & $\mathbf{5 . 0 7}$ & $\mathbf{4 . 1 1}$ & $\mathbf{0 . 0 0 0 2}$ & 3.95 & $\mathbf{3 . 4 6}$ & $\mathbf{4 . 1 2}$ & $\mathbf{0 . 0 0 1 5}$ \\
GOAL/SMT & 4.86 & $\mathbf{5 . 4 4}$ & $\mathbf{4 . 6 6}$ & $\mathbf{0 . 0 0 0 4}$ & 4.71 & 4.32 & 4.85 & 0.0850 \\
CMPT & 4.26 & $\mathbf{5 . 0 2}$ & $\mathbf{3 . 9 9}$ & $\mathbf{0 . 0 0 0 0}$ & 3.40 & 3.22 & 3.47 & 0.3335 \\
SCOMP & 3.81 & $\mathbf{4 . 5 7}$ & $\mathbf{3 . 5 5}$ & $\mathbf{0 . 0 0 0 0}$ & 2.75 & 2.91 & 2.70 & 0.5965 \\
SLEARN & 3.92 & $\mathbf{4 . 3 5}$ & $\mathbf{3 . 7 7}$ & $\mathbf{0 . 0 2 4 3}$ & 3.10 & 3.55 & $\mathbf{2 . 9 4}$ & $\mathbf{0 . 0 0 5 2}$ \\
COOP & 3.78 & $\mathbf{4 . 8 9}$ & $\mathbf{3 . 3 9}$ & $\mathbf{0 . 0 0 0 0}$ & 3.08 & $\mathbf{3 . 5 4}$ & $\mathbf{2 . 9 2}$ & $\mathbf{0 . 0 0 5 3}$ \\
\hline
\end{tabular}

\subsubsection{Persuasive Feature Effect Based on Within-Group Comparison}

In this subsection, we present the within-group comparisons at the overall and cultural group levels.

Overall Within-Group Comparison: Table 4 shows the posthoc pairwise comparisons based on the rating and ranking measures for the overall population sample. For the most part, in each pair, the left-side feature has a higher value than the right-side feature. The pairwise comparisons show that there is a significant difference $(p<0.05)$ between each pair of features based on the rating measure, except for reward-competition, social learning-social comparison, social learning-cooperation and social comparison-cooperation. The last three exceptions are applicable to the ranking measure as well. In particular, goal-setting/self-monitoring, regardless of measure, is significantly higher than social learning, social comparison and cooperation $(p<0.001)$.

Table 4. Posthoc pairwise comparisons of persuasive features for the overall population sample.

"-" means there is no significant difference between each pair of features; bold values indicate the result of pairwise comparison is in the same direction regardless of measure and culture; REWD = Reward, GOAL/SMT = Goal-Setting/Self-Monitoring, CMPT = Competition, SCOMP = Social Comparison, SLEARN = Social Learning, COOP = Cooperation.

\begin{tabular}{ccccccc}
\hline & \multicolumn{3}{c}{ Rating Measure } & \multicolumn{3}{c}{ Ranking Measure } \\
\hline Feature Comparison & Score1 & Score2 & $p$-Value & Score1 & Score2 & $p$-Value \\
\hline GOAL/SMT-REWD & 4.86 & 4.37 & 0.0062 & 4.71 & 3.95 & 0.0001 \\
GOAL/SMT-CMPT & 4.86 & 4.26 & 0.0010 & 4.71 & 3.40 & 0.0001 \\
GOAL/SMT-SLEARN & $\mathbf{4 . 8 6}$ & $\mathbf{3 . 9 2}$ & $\mathbf{0 . 0 0 0 1}$ & $\mathbf{4 . 7 1}$ & $\mathbf{3 . 1 0}$ & $\mathbf{0 . 0 0 0 1}$ \\
GOAL/SMT-SCOMP & $\mathbf{4 . 8 6}$ & $\mathbf{3 . 8 1}$ & $\mathbf{0 . 0 0 0 1}$ & $\mathbf{4 . 7 1}$ & $\mathbf{2 . 7 5}$ & $\mathbf{0 . 0 0 0 1}$ \\
GOAL/SMT-COOP & $\mathbf{4 . 8 6}$ & $\mathbf{3 . 7 8}$ & $\mathbf{0 . 0 0 0 1}$ & $\mathbf{4 . 7 1}$ & $\mathbf{3 . 0 8}$ & $\mathbf{0 . 0 0 0 1}$ \\
REWD-CMPT & 4.37 & 4.26 & - & 3.95 & 3.40 & 0.0014 \\
REWD-SLEARN & 4.37 & 3.92 & 0.0001 & 3.95 & 3.10 & 0.0010 \\
REWD-SCOMP & 4.37 & 3.81 & 0.0010 & 3.95 & 2.75 & 0.0010 \\
REWD-COOP & 4.37 & 3.78 & 0.0001 & 3.95 & 3.08 & 0.0001 \\
CMPT-SLEARN & 4.26 & 3.92 & 0.0020 & 3.40 & 3.10 & - \\
CMPT-SCOMP & 4.26 & 3.81 & 0.0001 & 3.40 & 2.75 & 0.0001 \\
CMPT-COOP & 4.26 & 3.78 & 0.0100 & 3.40 & 3.08 & - \\
SLEARN-SCOMP & 3.92 & 3.81 & - & 3.10 & 2.75 & - \\
SLEARN-COOP & 3.92 & 3.78 & - & 3.10 & 3.08 & - \\
SCOMP-COOP & 3.81 & 3.78 & - & 2.75 & 3.08 & - \\
\hline
\end{tabular}

Collectivist Within-Group Comparison: Table 5 shows the posthoc pairwise comparisons for the collectivist culture. There is a significant difference between over $45 \%$ and $30 \%$ of the pairs of features based on rating and ranking measures, respectively. For example, regardless of measure, just as we saw in the overall within-group comparison, there is a significant difference $(p<0.05)$ between goal-setting/self-monitoring, on one hand, and social learning, social comparison and cooperation, on the 
other hand. However, the significant difference between goal-setting/self-monitoring and social learning is marginal $(p=0.0768)$.

Table 5. Posthoc pairwise comparisons of persuasive features for the collectivist population sample. "-" means there is no significant difference between each pair of features; bold values indicate the result of pairwise comparison is in the same direction regardless of measure and culture; italicized values indicate marginally significant comparisons; REWD = Reward, GOAL/SMT = Goal-Setting/Self-Monitoring, $\mathrm{CMPT}=$ Competition, $\mathrm{SCOMP}=$ Social Comparison, $\mathrm{SLEARN}=$ Social Learning, $\mathrm{COOP}=$ Cooperation .

\begin{tabular}{ccccccc}
\hline & \multicolumn{3}{c}{ Rating Measure } & \multicolumn{3}{c}{ Ranking Measure } \\
\hline Feature Comparison & Score1 & Score2 & $p$-Value & Score1 & Score2 & $p$-Value \\
\hline GOAL/SMT-REWD & 5.44 & 5.07 & - & 4.32 & 3.46 & 0.0328 \\
GOAL/SMT-CMPT & 5.44 & 5.02 & - & 4.32 & 3.22 & 0.0018 \\
GOAL/SMT-SLEARN & $\mathbf{5 . 4 4}$ & $\mathbf{4 . 3 5}$ & $\mathbf{0 . 0 0 0 1}$ & $\mathbf{4 . 3 2}$ & $\mathbf{3 . 5 5}$ & $\mathbf{0 . 0 7 6 8}$ \\
GOAL/SMT-SCOMP & $\mathbf{5 . 4 4}$ & $\mathbf{4 . 5 7}$ & $\mathbf{0 . 0 0 0 1}$ & $\mathbf{4 . 3 2}$ & $\mathbf{2 . 9 1}$ & $\mathbf{0 . 0 0 0 1}$ \\
GOAL/SMT-COOP & $\mathbf{5 . 4 4}$ & $\mathbf{4 . 8 9}$ & $\mathbf{0 . 0 1 0 1}$ & $\mathbf{4 . 3 2}$ & $\mathbf{3 . 5 4}$ & $\mathbf{0 . 0 6 8 4}$ \\
REWD-CMPT & 5.07 & 5.02 & - & 3.46 & 3.22 & - \\
REWD-SLEARN & 5.07 & 4.35 & 0.0002 & 3.46 & 3.55 & - \\
REWD-SCOMP & 5.07 & 4.57 & 0.0176 & 3.46 & 2.91 & - \\
REWD-COOP & 5.07 & 4.89 & - & 3.46 & 3.54 & - \\
CMPT-SLEARN & 5.02 & 4.35 & 0.0007 & 3.22 & 3.55 & - \\
CMPT-SCOMP & 5.02 & 4.57 & 0.0517 & 3.22 & 2.91 & - \\
CMPT-COOP & 5.02 & 4.89 & - & 3.22 & 3.54 & - \\
SLEARN-SCOMP & 4.35 & 4.57 & - & 3.55 & 2.91 & - \\
SLEARN-COOP & 4.35 & 4.89 & - & 3.55 & 3.54 & - \\
SCOMP-COOP & 4.57 & 4.89 & - & 2.91 & 3.54 & - \\
\hline
\end{tabular}

Individualist Within-Group Comparison: Table 6 shows the posthoc pairwise comparisons for the individualist culture. There is a significant difference between most of the pairs of features, except for a few. Over $65 \%$ and $85 \%$ of the rating- and ranking-based pairwise comparisons, respectively, with the left-sided feature having a higher value than the right-sided value, are statistically significant $(p<0.05)$. For example, regardless of measure, just as we saw in the overall and collectivist within-group comparisons, there is a significant difference $(p<0.001)$ between goal-setting/self-monitoring, on one hand, and social learning, social comparison and cooperation, on the other hand. In addition, regardless of measure, there is a significant difference $(p<0.05)$ between goal-setting/self-monitoring, on one hand, and reward and competition, on the other hand. Similarly, regardless of measure, there is a significant difference $(p<0.05)$ between reward, on one hand, and social learning, social comparison and cooperation, on the other hand. Finally, regardless of measure, there is a significant difference $(p<0.01)$ between competition, on one hand, and social comparison and cooperation, on the other hand. 
Table 6. Posthoc pairwise comparisons of persuasive features for the individualist population sample. "_" means there is no significant difference between each pair of features; bold values indicate the result of pairwise comparison is in the same direction regardless of measure and culture; REWD = Reward, GOAL/SMT = Goal-Setting/Self-Monitoring, CMPT = Competition, SCOMP = Social Comparison, SLEARN $=$ Social Learning, $\mathrm{COOP}=$ Cooperation .

\begin{tabular}{ccccccc}
\hline & \multicolumn{3}{c}{ Rating Measure } & \multicolumn{3}{c}{ Ranking Measure } \\
\hline Feature Comparison & Score1 & Score2 & $p$-Value & Score1 & Score2 & $p$-Value \\
\hline GOAL/SMT-REWD & 4.66 & 4.11 & 0.0122 & 4.85 & 4.12 & 0.0001 \\
GOAL/SMT-CMPT & 4.66 & 3.99 & 0.0001 & 4.85 & 3.47 & 0.0001 \\
GOAL/SMT-SLEARN & $\mathbf{4 . 6 6}$ & $\mathbf{3 . 7 7}$ & $\mathbf{0 . 0 0 0 1}$ & $\mathbf{4 . 8 5}$ & $\mathbf{2 . 9 4}$ & $\mathbf{0 . 0 0 0 1}$ \\
GOAL/SMT-SCOMP & $\mathbf{4 . 6 6}$ & $\mathbf{3 . 5 5}$ & $\mathbf{0 . 0 0 0 1}$ & $\mathbf{4 . 8 5}$ & $\mathbf{2 . 7 0}$ & $\mathbf{0 . 0 0 0 1}$ \\
GOAL/SMT-COOP & $\mathbf{4 . 6 6}$ & $\mathbf{3 . 3 9}$ & $\mathbf{0 . 0 0 0 1}$ & $\mathbf{4 . 8 5}$ & $\mathbf{2 . 9 2}$ & $\mathbf{0 . 0 0 0 1}$ \\
REWD-CMPT & 4.11 & 3.99 & - & 4.12 & 3.47 & 0.0006 \\
REWD-SLEARN & 4.11 & 3.77 & 0.0139 & 4.12 & 2.94 & 0.0001 \\
REWD-SCOMP & 4.11 & 3.55 & 0.0001 & 4.12 & 2.70 & 0.0001 \\
REWD-COOP & 4.11 & 3.39 & 0.0001 & 4.12 & 2.92 & 0.0001 \\
CMPT-SLEARN & 3.99 & 3.77 & - & 3.47 & 2.94 & 0.0105 \\
CMPT-SCOMP & 3.99 & 3.55 & 0.0022 & 3.47 & 2.70 & 0.0001 \\
CMPT-COOP & 3.99 & 3.39 & 0.0001 & 3.47 & 2.92 & 0.0066 \\
SLEARN-SCOMP & 3.77 & 3.55 & - & 2.94 & 2.70 & - \\
SLEARN-COOP & 3.77 & 3.39 & - & 2.94 & 2.92 & - \\
SCOMP-COOP & 3.55 & 3.39 & - & 2.70 & 2.92 & - \\
\hline
\end{tabular}

\subsection{Overall and Culture-Specific Persuasion Profiles}

Table 7 shows the persuasion profiles of the overall and culture-specific groups. They were ordered from the most persuasive feature to the least persuasive feature, which was based on either the statistically significant or numerical differences between proximal pairs. Overall and regardless of culture and measure, users were more likely to be susceptible to goal-setting/self-monitoring than the other features. Moreover, based on the rating measure, the collectivist group was more likely to be susceptible to all six persuasive features than the individualist group. However, based on the ranking measure, the individualist group was more likely to be susceptible to goal-setting/self-monitoring and reward than the collectivist group. On the other hand, the collectivist group was more likely to be susceptible to cooperation and social learning than the individualist group. We discussed the implications of these findings in the context of tailoring in the discussion section.

Table 7. Persuasion profiles based on the perceived persuasiveness of strategies for the overall and culture-specific samples; COL = Collectivist culture, IND = Individualist culture; "the underlined" indicates where IND and COL significantly differ $(p<0.05)$ with respect to each measure, with the bolder feature indicating higher users' susceptibility; “*” indicates marginal significant difference $(p=0.085)$ between the concerned pair of features.

\begin{tabular}{ccc}
\hline Sample & Measure & Ordered List of Persuasive Features from Most to Least Persuasive \\
\hline \multirow{2}{*}{ Overall } & Rating & Goal/SMT, Reward, Compete, Social Learn, Compare, Cooperate \\
& Ranking & Goal/SMT, Reward, Compete, Social Learn, Cooperate, Compare \\
COL & Rating & Goal/SMT, Reward, Compete, Cooperate, Compare, Social Learn \\
& Ranking & Goal/SMT $*$ Social Learn, Cooperate, Reward, Compete, Compare \\
IND & Rating & Goal/SMT, Reward, Compete, Social Learn, Compare, Cooperate \\
& Ranking & Goal/SMT ${ }^{*}$, Reward, Compete, Social Learn, Cooperate, Compare \\
\hline
\end{tabular}

\section{Discussion}

We presented the persuasion profiles of our target populations based on six commonly employed persuasive features in fitness applications on the market. Specifically, we presented the level of 
susceptibility of the overall population sample and culture-based groups to goal-setting/self-monitoring, reward, competition, social comparison, social learning and cooperation. Our analysis of variance presented in Section 5.4 showed that there was an interaction between culture and persuasive feature. Table 7 shows the persuasion profiles for the respective cultural groups, which was based on the between-group analysis results shown in Table 3. In the between-group analysis results, out of the 12 between-group comparisons (six ratings and six rankings), nine were consistent with expectations and only three were inconsistent. Individualist participants were expected to score higher in goal-setting/self-monitoring and reward, while collectivist participants in the other features. Though a number of the group differences were not statistically significant, the numerical differences were in the expected directions. For example, cooperation and social learning were significantly rated and ranked higher by the collectivist group than the individualist group (four consistencies). Social comparison was rated and ranked higher by the collectivist group than the individualist group, though the difference was not statistically significantly (two consistencies). Competition, in particular, was significantly rated higher by the collectivist group than the individualist group (one consistency). Though competition was ranked higher by the individualist group than by the collectivist group (one inconsistency), the difference was neither substantial $(0.25)$ nor significant at $p<0.05$. Moreover, the individualist group ranked goal-setting/self-monitoring and reward marginally and significantly, respectively, higher than the collectivist group as expected (two consistencies). The only unexpected inconsistency, with respect to the personal features, was the rating of goal-setting/self-monitoring and reward by the collectivist group higher than by the individualist group (two inconsistencies). We explained these results in detail (in the context of personal and social features) and inconsistencies in the rest of the discussion.

\subsection{Users' Susceptibility to Personal Features}

Overall, irrespective of culture, the participants were more likely to be susceptible to goal-setting/self-monitoring based on the raring and ranking measures. This finding might not be surprising given that a health application such as a fitness application aimed at behavior change might not be considered useful by potential users if it does not have the capability of goal-setting and self-monitoring. In our prior study [17], we argued that every minimally viable health application aimed at behavior change, for it to be effective, must have at least the functionality of goal-setting and self-monitoring, both of which we considered complimentary fundamental features. We considered them complimentary features that must be implemented side by side in a fitness application because it is no use setting goals if the user of the application cannot track his/her behavior towards achieving the set goal. This means if a user sets goals, then s/he should be given the opportunity to track his progress towards reaching those goals as well. Similarly, if a user is allowed to track his/her behavior, then s/he should be given the opportunity to set goals to achieve the target behavior [17]. For this reason, in our storyboards, we intentionally implemented goal-setting and self-monitoring as a composite feature because we viewed them as complementary. Thus, when combined with self-monitoring, goal-setting provides the user with the opportunity to set a target goal and track his/her activities towards reaching the target goal through self-monitoring. The finding that users were most likely to be susceptible to goal-setting/self-monitoring, regardless of culture, could be said to be consistent with the prior finding by Oyibo et al. [25], whose studies on persuasion profiles were based on the six Cialdini's [24] principles of persuasion: commitment, reciprocity, authority, liking, consensus and scarcity. The authors found that, regardless of culture and gender [26], users were more likely to be susceptible to commitment. They mapped the Cialdini's commitment principle of persuasion to goal-setting as a way of operationalizing it in the persuasive technology domain. They explained that goal-setting in persuasive applications is equivalent to making a commitment to the application to achieve a certain goal set for oneself [25]. They put it in this way, by setting goals, especially "SMART" (specific, measurable, attainable, relevant and time-bound) goals, "the user is indirectly making a commitment to the persuasive system. As a result, the likelihood of the user performing the target behavior is higher than when no goal is set" [17]. Oinas-Kukkonen and Harjumaa [36] postulated that if persuasive systems support the making of 
commitments, which we operationalized as goal-setting (and self-monitoring), users will be more likely persuaded to engage in the target behavior.

Secondly, in our analysis (see Table 7), we found that, overall and especially for the individualist culture, reward was the second most persuasive (personal) feature to which users were susceptible. For the overall and individualist groups, reward was rated as more persuasive than the other features. Apart from goal-setting/self-monitoring and competition, the difference between reward and the other features was significant at $p<0.05$. Similarly, reward was ranked by the overall population and the individualist group as more persuasive than the other features apart from goal-setting/self-monitoring. For the collectivist group, reward was only rated as more persuasive than social learning and social comparison. In general, based on the rating and ranking measures, it is either reward was rated and/or ranked significantly higher than the other features (apart from goal-setting/self-monitoring) or the difference between reward's rating/ranking and the other features' rating/ranking is not statistically significant $(p>0.05)$. This finding is regardless of the cultural group you are looking at. As such, without considering the moderating effect of culture, reward could be said to be the second most persuasive feature to which users are more likely to be susceptible. Thus, in a basic fitness application aimed at changing health behaviors, reward should be implemented in concert with goal-setting/self-monitoring to amplify its motivational capability. Oyibo et al. [25] recommended that one way of making goal-setting/self-monitoring more effective in changing behavior is by accompanying it with reward. They argued that by providing users with incentives (e.g., points, badges, etc.) for accomplishing their set goals, users are able to visualize in concrete terms the short-term benefit of their target behavior [37]. This is important because most health benefits, especially with respect to exercise, may not be immediately visible - they may be long-term and/or not physical if not vigorously and consistently performed for a long time (e.g., push-up). Thus, to encourage users, there may be a need to provide them with concrete, short-term non-health benefits as a way to reinforce the performance of the target behavior [38,39].

\subsection{Users' Susceptibility to Social Features}

Overall, based on the rating and ranking measures, among the social features, participants are more likely to be susceptible to competition (see Table 4) than to social comparison, social learning and cooperation. Specifically, based on the rating measure, the mean rating of competition is significantly higher than the mean ratings of the other three social features $(p<0.01)$. Moreover, based on the ranking measure, the mean rating of competition is only significantly higher than the mean rating of social comparison $(p<0.01)$. Despite the fact that the mean ranking of competition is numerically higher than the mean ranking of cooperation/social learning, there is no significant difference between them $(p>0.05)$. That said, given that the mean rating of competition is significantly higher than the mean rating of the other features, we recommend that, in a one-size-fits-all social fitness application, the competition feature should be given priority over social learning, social comparison and cooperation.

For the individualist culture, in particular, competition should be given priority over the others. As we saw in the rating measure and ranking measure (Figure 3), users from the individualist culture are only likely to be susceptible to competition among the social features. Specifically, we saw that they rated and ranked the other three features way below the neutral value of 4 and the mean value of 3.5, respectively, which is an indication that they might not be susceptible to social learning, social comparison and cooperation. This finding is consistent with the prior finding by Oyibo et al. [27,37] in the non-domain-specific context of persuasive technology. The authors found that individualist users (Canadians) were more likely to be susceptible to competition than social learning and social comparison.

On the other hand, based on the rating measure (Figure 3), we saw that users from the collectivist culture are likely to be susceptible to all four social features as they rated them way above the neutral value of 4 . Moreover, the ranking measure indicates that the collectivist users are more likely to be susceptible to social learning and cooperation (mean ranking equal to or above the mean value of 3.5) than competition and social comparison (mean ranking less than the mean value of 3.5). Thus, we recommend 
that, in implementing a socially oriented fitness application for the collectivist group, social learning and cooperation should be given priority over competition and social comparison.

\subsection{Differences between Collectivist and Individualist Cultures}

Our two-way analysis of variance showed that there was an interaction between culture and persuasive feature based on the ranking measure. The between-group comparison results (shown in Table 3) suggest that: (1) individualist culture is more likely to be susceptible to reward $(p<0.01)$ and goal-setting/self-monitoring than the collectivist culture ( $p=0.0850-$ marginal). However, the collectivist culture is more likely to be susceptible to cooperation $(p<0.01)$ and social learning than the individualist culture $(p<0.01)$. In particular, this finding supports the rating-based finding, in which the collectivist group rated cooperation $(p<0.000)$ and social learning $(p<0.05)$ significantly higher than the individualist group.

Overall, by accounting for the possible influence of culture in the rating of the perceived persuasiveness of the storyboards, we based the conclusions of our analysis on the ranking of the persuasive features by the two cultural groups. Research [14] shows that culture influences the level of criticality in the judgment of HCI artifacts. Moreover, we argued that in the rating of the storyboards in terms of the perceived persuasiveness of the illustrated features, participants must have taken into consideration the UI design of the storyboards. For this reason, the individualist participants (Canadians/Americans) were more likely to be critical of the UI design of the storyboards than the collectivist participants (Nigerians) given that the former were more technologically advanced, tech-savvy and have higher mobile Internet experience. In particular, previous research [40] shows that users with higher Internet experiences are more likely to be critical of HCI artifacts when evaluating them. In our case, Table 2 shows that, overall, the individualist group had higher Internet experience than the collectivist group. For example, only $37 \%$ of the Nigerian subjects (collectivist group) had over 10 years of Internet experience, compared with $85 \%$ of the Canadian/American subjects (individualist group). Moreover, $29 \%$ of the individualist participants had over 20 years of Internet experience, compared with $0 \%$ of the collectivist participants. For these reasons, we based our conclusions of our analysis specifically on the ranking of the persuasive features (shown in Table 3) as follows: (1) the individualist culture is more likely to be susceptible to personal features than the collectivist culture; and (2) the collectivist culture is more likely to be susceptible to social features than the individualist culture.

One plausible explanation for why the individualist culture is more likely to be susceptible to personal features than the collectivist culture, while the collectivist culture is more likely to be susceptible to social features than the individualist culture can be found in Hofstede's [30] cultural framework. The framework defines individualist culture as that type of culture in which people are independent and self-motivated in the pursuit of life's goals. On the other hand, it defines the collectivist culture as that type of culture in which people are interdependent and thus work together to achieve the collective goal of the in-group to which they belong. In our study, our two cultural groups exemplify these theoretical underpinnings. Canada and United States, which make up the individualist group, are typical examples of individualist countries in the West. On the other hand, Nigeria, the collectivist group is a typical example of a collectivist country in Africa. Prior research in the theoretical domain of behavior change has found that the physical activity behavior of individualist and collectivist cultures are driven by personal and social factors, respectively. For example, in the physical activity domain, Oyibo et al. $[7,41]$ found that self-efficacy and self-regulation are the strongest drivers of physical activity in the individualist culture, while social support is the strongest driver of physical activity in the collectivist culture. These theoretical determinants of behavior were mapped by the authors to implementable persuasive strategies in the application domain. Specifically, self-efficacy and self-regulation were mapped to personal strategies such as goal-setting/self-monitoring and reward, while social support was mapped to social strategies such as cooperation, social comparison and social 
learning. The findings of our current study, especially with respect to the individualist culture, tend to replicate the prior findings in the theoretical domain of behavior change [7,41].

Furthermore, a plausible explanation for why the individualist group is less likely to be susceptible to social features, compared with the collectivist group, is the concern about privacy and confidentiality. Previous studies (e.g., [12]) have shown that people in individualist cultures express great concerns about the privacy and confidentiality of their health information. Thus, in our current study, we saw that they are less likely to be responsive to social strategies, which may involve other users having access to their health information with respect to the performance and non-performance of the target behavior.

\subsection{Overall and Culture-Specific Persuasive Design Guidelines}

In our study, we uncovered some key findings in the overall and culture-specific persuasion profiles. We also found some significant differences between the individualist and collectivist groups. We summarize the key findings and significant differences between both cultural groups in the form of design guidelines for developing persuasive health applications, especially in the physical activity domain. They include the following:

1. In a one-size-fits-all fitness application, goal-setting/self-monitoring should be given priority over other persuasive features, followed by reward. In other words, personal strategies should be given priority over social features in the development of fitness applications targeted at users in both types of culture, with goal-setting/self-monitoring and reward implemented in concert.

2. In individualist cultures, personal features, such as goal-setting/self-monitoring and reward, should be employed in motivating behavior change. If social features are to be employed, then competition should be given priority over cooperation, social comparison and social learning.

3. In collectivist cultures, both personal and social features could be employed in changing behavior. However, in a social context, cooperation and social learning should be given priority over competition and social comparison given that members of collectivist cultures tend to work together rather than against one another to achieve collective goals.

\subsection{Contributions and Future Work}

The main contribution of study is two-fold: expanding the body of knowledge and provision of empirical evidence for the design of fitness apps in the application domain. Regarding the expansion of the body of knowledge, our paper validated culture-specific design guidelines (based on theoretical determinants of physical activity behavior) in the persuasive technology domain. Using social cognitive theory, Oyibo et al. [7,41] had found that (1) self-efficacy and self-regulation (mapped to goal-setting, self-monitoring, reward, etc.) were the strongest determinants of physical activity behavior for the individualist culture; and (2) social support was the strongest determinant of physical activity behavior for the collectivist culture. Thus, they concluded that personal factors are more likely to be effective in changing behavior among individualist users than among collectivist users, while social factors are more likely to be effective in changing behavior among collectivist users than individualist users. Our current study was able to replicate and validate this prior finding based on the ranking of the persuasive features we investigated. Specifically, based on the ranking measure, we confirmed in the application domain that personal persuasive features (such as goal-setting/self-monitoring and reward) are more likely to be effective in changing physical activity behavior among users in the individualist culture than among users in the collectivist culture. Moreover, we confirmed in the application domain that social persuasive features (such as cooperation and social learning) are more likely to be effective in changing physical activity behavior among users in the collectivist culture than among users in the individualist culture. In our future work, we hope to investigate these findings in the application domain. Specifically, based on the current culture-specific findings, we hope to design and implement two versions of a fitness application (personal and social) for both cultures. Then, 
we would evaluate both versions within and across cultures to uncover how our current findings generalize to the application domain (see Oyibo et al. [42] for the full description of the application and study design).

Regarding our second contribution in the application domain, while it can be argued that the big players (such as Samsung, Google, Apple, Fitbit, etc.) in the fitness domain could utilize Big Data analytics to personalize fitness apps at the individual level, this is may not be the case with small players in the field, which may lack the required resources (time, cost and programming expertise) for individual-level Big-Data driven personalization. Moreover, big players such as Fitbit utilize wearables such as Fitbit trackers and bands and commercialize their services [43], which low-income users, especially in Africa, may not be able to afford. The knowledge our paper contributes to the literature will benefit small players, who are independent of and not as sophisticated as the big players, in the development of evidence-based tailored fitness apps for their culturally diverse target users.

\subsection{Limitations}

Our study has a number of limitations. The primary limitation of our study is that it is based on users' perceived persuasiveness of the persuasive features illustrated on storyboards, which mocked up a fitness application. Thus, our overall and culture-specific findings might not generalize to the application domain, in which users from both types of culture might have to use an actual fitness application in the wild. Second, our storyboards did not exemplify as much as possible the actual visual and user experience design of the fitness application prototype we envisaged in the future due to limited resources on our part at the time. This might have impacted the responses of participants across cultures. However, our current findings sets the stage for a more practical, real-life application design in the future, which we intended to evaluate in the wild in both types of culture. Third, our culture-specific findings were based on participants whose countries of origin and residence are Canada/United States and Nigeria. As such, our findings might not generalize to other individualist and collectivist cultures. Hence, there is a need to conduct similar studies in other individualist and collectivist cultures as well in the future to test the generalizability of our findings. A fourth limitation of our findings is that we did not randomize the order of presentation of the storyboards, which could have affected our findings. Finally, we did not take the current physical activity level of participants into consideration in our analysis. This is another limitation of our study, which could have had an effect on our culture-specific findings with respect to how participants rated and ranked the persuasive features in terms of perceived persuasiveness. Future work can investigate this as well.

\section{Conclusions}

We presented the persuasion profiles of users in individualist and collectivist cultures and the differences that exist between them using Canada/United States and Nigeria as a case study. Using rating and ranking measures, we investigated their different levels of susceptibility to six commonly employed persuasive features in fitness applications on the market. The features include goal-setting/self-monitoring, reward, competition, cooperation, social comparison and social learning, all of which were illustrated on storyboards. Our results showed that, overall-based on the rating measure-users were more likely to be susceptible to personal features (goal-setting/self-monitoring and reward) than social features (competition, cooperation, social comparison and social learning). Thus, we recommend that, in a one-size-fits-all fitness application, goal-setting/self-monitoring should be given priority over other persuasive features, followed by reward. More specifically, our results showed that, based on the rating measure, users, in the individualist culture, are only likely to be susceptible to one social feature (competition) in addition to the two personal features (goal-setting/self-monitoring and reward). However, in the collectivist culture, users are likely to be susceptible to both personal and social features. Finally, based on the ranking measure, our results showed that the individualist culture was more likely to be susceptible to personal features (goal-setting/self-monitoring and reward) than the collectivist culture. However, based on the ranking measure, the collectivist culture was more likely to 
be susceptible to social features (cooperation and social learning) than the individualist culture. In future work, we aim to validate these findings in an actual fitness application, in which the personal and social features were implemented and evaluated in the wild.

Author Contributions: Conceptualization, K.O. and J.V.; methodology, K.O.; software, NA; validation, K.O. and J.V.; formal analysis, K.O.; investigation, K.O.; resources, J.V.; data curation, K.O.; writing-original draft preparation, K.O.; writing-review and editing, K.O. and J.V.; visualization, K.O.; supervision, J.V.; project administration, J.V.; funding acquisition, J.V.

Funding: This research was funded by the Canadian Government and the University of Saskatchewan (Grant no. RGPIN-2016- 05762). The second author received the Natural Sciences and Engineering Research Council of Canada Discovery Grant, while the first author received the Saskatchewan Innovation and Opportunity Scholarship.

Conflicts of Interest: The authors declare no conflict of interest.

\section{References}

1. Oyibo, K.; Vassileva, J. Investigation of the Moderating Effect of Race-Based Personalization of Behavior Model Design in Fitness Application. SN Appl. Sci. 2019. [CrossRef]

2. Oyibo, K.; Adaji, I.; Orji, R.; Olabenjo, B.; Azizi, M.; Vassileva, J. Perceived Persuasive Effect of Behavior Model Design in Fitness Apps. In Proceedings of the 26th Conference on User Modeling, Adaptation and Personalization, Singagpore, 8-11 July 2018; pp. 219-228.

3. Kaptein, M.; De Ruyter, B. Adaptive Persuasive Systems: A Study of Tailored Persuasive Text Messages to Reduce Snacking. ACM Trans. Interact. Intell. Syst. 2012, 2, 1-25. [CrossRef]

4. Kaptein, M.; Markopoulos, P.; De Ruyter, B.; Aarts, E. Personalizing persuasive technologies: Explicit and implicit personalization using persuasion profiles. Int. J. Hum. Comput. Stud. 2015, 77, 38-51. [CrossRef]

5. Oyibo, K.; Ali, Y.S.; Vassileva, J. Gender Difference in the Credibility Perception of Mobile Websites: A Mixed Method Approach. In Proceedings of the 2016 Conference on User Modeling Adaptation and Personalization-UMAP '16, Halifax, NS, Canada; 2016; pp. 75-84.

6. Cyr, D.; Head, M.; Ivanov, A. Perceptions of Mobile Device Website Design: Culture, Gender and Age Comparisons. In Web Technologies: Concepts, Methodologies, Tools, and Applications; IGI Global: Hershey, PA, USA, 2009; pp. 173-200. [CrossRef]

7. Oyibo, K.; Orji, R.; Vassileva, J. Developing Culturally Relevant Design Guidelines for Encouraging Physical Activity: A Social Cognitive Theory Perspective. J. Healthc. Inform. Res. 2018, 1-34. [CrossRef]

8. Sun, H. Building a Culturally-Competent Corporate Web Site: An Exploratory Study of Cultural Markers in Multilingual Web Design. In Proceedings of the 19th Annual International Conference on Computer Documentation, Sante Fe, NM, USA, 21-24 October 2001; pp. 95-102. [CrossRef]

9. Orji, R.; Moffatt, K. Persuasive technology for health and wellness: State-of-the-art and emerging trends. Health Inform. J. 2016. [CrossRef] [PubMed]

10. Khaled, R. Culturally-Relevant Persuasive Technology. Available online: http://rillakhaled.com/research/ thesis.pdf (accessed on 31 December 2008).

11. Khaled, R.; Barr, P.; Noble, J.; Fischer, R.; Biddle, R. Our place or mine? Exploration into collectivism-focused persuasive technology design. In Proceedings of the First International Conference on Persuasive Technology for Human Well-Being, PERSUASIVE 2006, Eindhoven, The Netherlands, 18-19 May 2006; pp. 72-83.

12. Orji, R.; Oyibo, K.; Lomotey, R.K.; Orji, F.A. Socially-driven persuasive health intervention design: Competition, social comparison, and cooperation. Health Inform. J. 2018. [CrossRef] [PubMed]

13. Munson, S.; Consolvo, S. Exploring Goal-setting, Rewards, Self-monitoring, and Sharing to Motivate Physical Activity. In Proceedings of the 2012 6th International Conference on Pervasive Computing Technologies for Healthcare (PervasiveHealth) and Workshops, San Diego, CA, USA, 21-24 May 2012; pp. 25-32. [CrossRef]

14. Oyibo, K.; Ali, Y.S.; Vassileva, J. An empirical analysis of the perception of mobile website interfaces and the influence of culture. In Proceedings of the Personalized Persuasive Technology Workshop, Salzburg, Austria, 5 April 2016; pp. 44-56.

15. Hofstede, G.; Hofstede Geert, J. Cultures and Organizations: Software of the Mind; Mcgraw-hill: New York, NY, USA, 2005.

16. Karreman, J.; Romeo, P.; Li, Q. Cross-Cultural HCI and UX Design: A Comparison of Chinese and Western User Interfaces. 2016. Unpublished work. [CrossRef] 
17. Oyibo, K.; Adaji, I.; Vassileva, J. Susceptibility to Fitness App's Persuasive Features: Differences between Acting and Non-Acting Users. In Proceedings of the Adjunct Publication of the 27th Conference on User Modeling, Adaptation and Personalization, Larnaca, Cyprus, 9-12 June 2019.

18. Oinas-kukkonen, H.; Harjumaa, M. Persuasive Systems Design Key Issues, Process Model, and System Features. Commun. Assoc. Inf. Syst. 2009, 24, 485-500. [CrossRef]

19. Stibe, A. Exploring Social Influence on Customer Engagement: A Pilot Study on the Effects of Social Learning, Social Comparison, and Normative Influence. In Proceedings of the 2013 46th Hawaii International Conference on System Sciences, Wailea, Maui, HI, USA, 7-10 January 2012. [CrossRef]

20. Oyibo, K.; Vassileva, J. Investigation of Persuasive System Design Predictors of Competitive Behavior in Fitness Application: A Mixed Method Approach. Digit. Heal. 2019.

21. Kaptein, M.; Markopoulos, P. Can you be persuaded? individual differences in susceptibility to persuasion. In Proceedings of the 12th IFIP TC 13 International Conference, Uppsala, Sweden, 24-28 August 2009; pp. 115-118.

22. Orji, R. Persuasion and Culture: Individualism-Collectivism and Susceptibility to Influence Strategies. In Proceedings of the Personalized Persuasive Technology Workshop, Salzburg, Austria, 5 April 2016.

23. Orji, R.; Mandryk, R.L.; Vassileva, J. Gender, age, and responsiveness to cialdini's persuasion strategies. In Proceedings of the 10th International Conference, PERSUASIVE 2015, Chicago, IL, USA, 3-5 June 2015; pp. 147-159.

24. Cialdini, R.B. Influence: The Psychology of Persuasion; HarperCollins: New York, NY, USA, 2006.

25. Oyibo, K.; Adaji, I.; Orji, R.; Olabenjo, B.; Vassileva, J. Susceptibility to persuasive strategies: A comparative analysis of nigerians vs. Canadians. In Proceedings of the 26th Conference on User Modeling, Adaptation and Personalization, Singapore, 8-11 July 2018; pp. 229-238.

26. Oyibo, K.; Adaji, I.; Orji, R.; Vassileva, J. The Susceptibility of Africans to Persuasive Strategies: A Case Study of Nigeria. In Proceedings of the Personalization in Persuasive Technology Workshop, Persuasive Technology 2018, Waterloo, ON, Canada, 17 April 2018.

27. Oyibo, K.; Orji, R.; Vassileva, J. The influence of culture in the effect of age and gender on social influence in persuasive technology. In Proceedings of the UMAP 2017-Adjunct Publication of the 25th Conference on User Modeling, Adaptation and Personalization, Bratislava, Slovakia, 9-12 July 2017.

28. Oyibo, K.; Ifebude, B.; Adaji, I.; Vassileva, J. Investigation of the Perceived Persuasive Features of Fitness App: A Case Study of Nigeria. In Proceedings of the 4th International Workshop on Personalizng Persuasive Technologies, Limassol, Cyprus, 9-11 April 2019.

29. Drozd, F.; Lehto, T.; Oinas-Kukkonen, H. Exploring perceived persuasiveness of a behavior change support system: A structural model. In Proceedings of the 7th International Conference, PERSUASIVE 2012, Linköping, Sweden, 6-8 June 2012; pp. 157-168. [CrossRef]

30. Hofstede, G. Dimensionalizing Cultures: The Hofstede Model in Context. Online Read. Psychol. Cult. 2011, 2, 1-26. [CrossRef]

31. Dunn, T.J.; Baguley, T.; Brunsden, V. From alpha to omega: A practical solution to the pervasive problem of internal consistency estimation. Br. J. Psychol. 2014, 105, 399-412. [CrossRef] [PubMed]

32. Hinton, P.R.; McMurray, I.; Brownlow, C.; Cozens, B. SPSS Explained; Routledge: Abingdon-on-Thames, UK, 2014.

33. Wobbrock, J.O.; Findlater, L.; Gergle, D.; Higgins, J.J. The aligned rank transform for nonparametric factorial analyses using only anova procedures. In Proceedings of the SIGCHI Conference on Human Factors in Computing Systems, Vancouver, BC, Canada, 7-12 May 2011. [CrossRef]

34. Higgins, J.J.; Tashtoush, S. An aligned rank transform test for interaction. Nonlinear World 1994, 1, $201-211$.

35. Kay, M.; Wobbrock, J.O. Package “ARTool”. Available online: https://cran.r-project.org/web/packages/ ARTool/ARTool.pdf (accessed on 16 November 2019).

36. Oinas-kukkonen, H.; Harjumaa, M. A Systematic Framework for Designing and Evaluating Persuasive Systems. In Proceedings of the Third International Conference, PERSUASIVE 2008, Oulu, Finland, 4-6 June 2008; pp. 164-176.

37. Oyibo, K.; Orji, R.; Vassileva, J. Investigation of the Persuasiveness of Social Influence in Persuasive Technology and the Effect of Age and Gender. In Proceedings of the International Workshop on Persuasive Technology, CEUR, Amsterdam, The Netherlands, 4 April 2017. 
38. Oyibo, K.; Orji, R.; Vassileva, J. Investigation of the social predictors of competitive behavior and the moderating effect of culture. In Proceedings of the UMAP 2017-Adjunct Publication of the 25th Conference on User Modeling, Adaptation and Personalization, Bratislava, Slovakia, 9-12 July 2017; pp. 419-424.

39. Consolvo, S.; Everitt, K.; Smith, I.; Landay, J.A. Design requirements for technologies that encourage physical activity. In Proceedings of the SIGCHI Conference on Human Factors in Computing Systems, Montréal, QC, Canada, 22-27 April 2006; pp. 457-466. [CrossRef]

40. Oyibo, K.; Ifeoma, A.; Vassileva, J. The Influence of Internet Experience on the Judgment of Mobile Web Design. In Proceedings of the 32nd Human-Computer Interaction Conference, Belfast, UK, 4-6 July 2018.

41. Oyibo, K. Designing Culture-based Persuasive Technology to Promote Physical Activity among University Students. In Proceedings of the 24th Conference on User Modeling, Adaptation and Personalization (UMAP 2016), Halifax, NS, Canada, 13-17 July 2016; pp. 321-324.

42. Oyibo, K.; Olagunju, A.-H.; Olabenjo, B.; Adaji, I.; Vassileva, J. BEN’FIT: Design, Implementation and Evaluation of a Culture-Tailored Fitness App. In Proceedings of the Adjunct Publication of the 27th Conference on User Modeling, Adaptation and Personalization, Larnaca, Cyprus, 9-12 June 2019.

43. Visnji, M. How Fitbit Makes Money? Available online: https://revenuesandprofits.com/how-fitbit-makesmoney/ (accessed on 16 November 2019).

(C) 2019 by the authors. Licensee MDPI, Basel, Switzerland. This article is an open access article distributed under the terms and conditions of the Creative Commons Attribution (CC BY) license (http://creativecommons.org/licenses/by/4.0/). 